\title{
Bioinformation
}

\author{
www.bioinformation.net
}

Software

\section{MAP MUTATION: a program for analyzing mutations in protein sequences}

\author{
Tamanna Anwar ${ }^{1}$ and Asad U Khan ${ }^{1,2 *}$ \\ ${ }^{1}$ Distribution Information Sub Centre, ${ }^{2}$ Interdisciplinary Biotechnology Unit, Aligarh Muslim University, Aligarh 202002, \\ India; Asad U Khan* - Email: huzzi99@hotmail.com; Phone: +91-571-2723088; Fax: +91-571-2721776; \\ * Corresponding author \\ received February 08, 2006; accepted February 12, 2006; published online February 20, 2006
}

\begin{abstract}
:
Avian influenza is an infectious disease of birds caused by type-A strains of the influenza virus. The unprecedented spread of the highly pathogenic avian influenza type A is a threat to veterinary and human health. Influenza viruses continuously undergo mutations and they lack proofreading mechanism. Hence, they evolve to new forms of the virus. We describe MAP MUTATION (developed using PERL script) to quickly compare two strains and display mutational information and report specific positions where mutation has occurred.
\end{abstract}

Availability: The PERL script is available from the authors for non-commercial purposes.

Keywords: sequence; mutation; virus; PERL script; analysis

Background:

The avian influenza (bird flu) is an infectious disease of the birds, ranging from mild to severe form of the illness. Influenza viruses pose significant challenge to both human and animal health. Highly pathogenic avian influenza virus infection can result in huge economic loss. Viruses of low virulence are changed to highly pathogenic viruses when they are circulating in the poultry population due to high mutation rate in their genome. [1] During the 1983-1984 outbreaks in the United States, the virulence of H5N2 virus started low. However, within six months the mortality rate caused by the virus reached $90 \%$. [2] The influenza viruses mutate rapidly and it is important to estimate and predict the rate of mutation in these viruses. [3] We describe a PERL script, named MAP MUTATION, for identifying mutations in the rapidly evolving avian influenza virus. MAP MUTATION can compare sequences of varying lengths by padding the shorter sequences with asterisks $(*)$. The program is tested for H5N1 proteins (unpublished data).

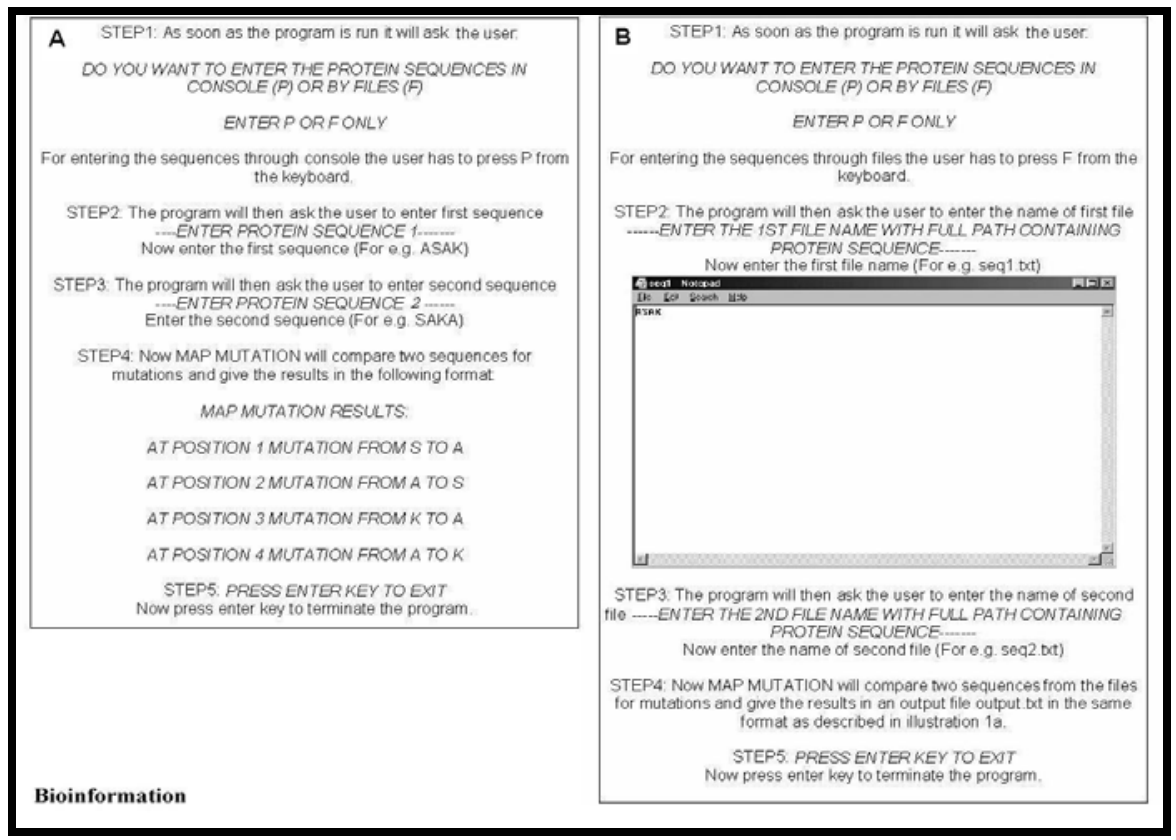

Figure 1: (A) Description of program flow while entering the input through console. (B) Description of program flow while entering the input through files 


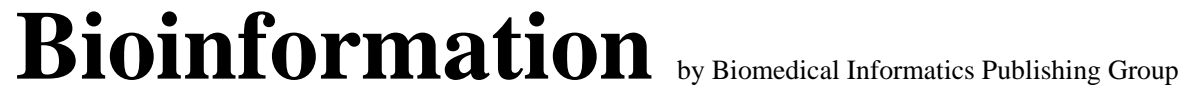

open access

\section{www.bioinformation.net}

\section{Software}

\begin{abstract}
Methodology:
Input:

MAP MUTATION is an interactive, user-friendly program for identifying mutations by comparing sequences of two different strains. MAP MUTATION accepts input sequences in the program console (for short sequences as shown in Figure 1A) or using TEXT file (as shown in Figure 1B using two different files for two sequences) containing the sequence (for longer sequences).
\end{abstract}

\section{Output:}

MAP MUTATION produces result instantly in the console when input is given in the console and the file output.txt is generated from sequences obtained through input files.

Caveats \& Future Development:
MAP MUTATION is a PERL script. A web based CGI (common gateway interface) is being developed for MAP MUTATION.

\section{Acknowledgement:}

The authors are grateful to Professor M. Saleemuddin for providing facilities to carryout this work and for his support throughout the project. BTIS is acknowledged for technical help. Department of Biotechnology, Ministry of Science and Technology, Government of India is acknowledged for the financial support.

\section{References:}

[1] S. Padhi, et al., Indian J Med Microbiol., 22:143 (2004) [Abstract]

[2] http://www.who.int/csr/don/2004_01_15/en/

[3] http://www.usatoday.com/news/health/2006-01-22bird-flu-mutations_x.htm

Edited by $P$. Kangueane

Citation: Anwar \& Khan, Bioinformation 1(3): 92-93 (2006)

License statement: This is an open-access article, which permits unrestricted use, distribution, and reproduction in any medium, for non-commercial purposes, provided the original author and source are credited. 